\title{
SELF-DIFFUSION IN UNIFORMLY DEFORMED $A-(C)$ INTERSTITIAL SOLID SOLUTIONS WITH CUBIC LATTICES
}

\author{
V. A. Volkov
}

UDC 539.219.3:548.4.001

Keywords: diffusion, interstitial solutions, octa- and tetra-interstices, vacancies.

Diffusion processes in solids are determined finally by interatomic interaction forces. One of the reasons for changing the interatomic interaction energy is crystal deformation. In the present work, the self-diffusion coefficient is calculated for uniformly deformed $A-(C)$ interstitial solid solutions with $\mathrm{BCC}$ and FCC lattices. Calculations are performed for vacancy models of diffusion theory $[1,2]$. In this model, the diffusion coefficient is determined as a ratio of flux density $j$ of isotopes of atom $A$ to the corresponding gradient of isotope concentration in the direction $i(i=x, y$, z) $\partial n / \partial i$ :

$$
D=-\frac{j}{\partial n / \partial i}
$$

It is considered that diffusion by hopping of atom $A$ can proceed only to the nearest vacant site, and the probability of transition per unit time is

$$
w=\frac{1}{\tau_{0}} e^{-\frac{\Delta u}{k T}},
$$

where $\tau_{0}$ is time on the order of the oscillation period corresponding to the maximum frequency in the acoustic spectrum of the crystal, $\Delta u$ is the height of the potential barrier separating the central atom and the neighboring vacancy. Designating the concentration of vacancies in the sites by $c_{v}$, we can write for the flux of atoms $A$, for example, in the direction of the $O x$ axis

$$
j_{x}=-w a_{x}^{2} c_{v} \frac{\partial n}{\partial x}
$$

(where $a_{x}$ is the lattice constant in the direction of the $O x$ axis). From here for the diffusion coefficient we obtain

$$
D_{x}=\frac{a_{x}^{2}}{\tau_{0}} c_{v} e^{-\frac{\Delta u}{k T}} .
$$

Thus, the problem is reduced to calculations of $c_{v}$ and $\Delta u$ in the deformed $A-(C)$ crystal.

Ural Federal University Named after the First President of Russia B. N. El'tsin, Ekaterinburg, Russia, e-mail: vavolk@mail.ru. Translated from Izvestiya Vysshikh Uchebnykh Zavedenii, Fizika, No. 2, pp. 109-112, February, 2013. Original article submitted December 29, 2011; revision submitted February 8, 2013. 
Let us consider the crystal with BCC lattice in which interstitial atoms $C$ occupy octa-interstices. The deformed state is assigned by the diagonal deformation tensor with components $\varepsilon_{1}, \varepsilon_{2}$, and $\varepsilon_{3}$. Then the lattice constants along the $O x, O y$, and $O z$ axes are written in the form

$$
a_{x}=a\left(1+\varepsilon_{1}\right), a_{y}=a\left(1+\varepsilon_{2}\right), a_{z}=a\left(1+\varepsilon_{3}\right),
$$

where $a$ the lattice constant for the non-deformed crystal. In this case, octa- and tetra-interstices in BCC solutions are split into positions of three types with energies $u_{i}^{(0)}$ and $u_{i}^{(T)}(i=1,2,3)$. In FCC crystals, interstices do not split after deformation, only the energies of octa- and tetra-interstices are shifted [3].

The equilibrium vacancy concentration for a preset temperature of the solution can be found from the extremum conditions for free energy $F$ of the system [2-4] that allow the vacancy concentration $c_{v}^{(0)}$ for atoms $C$ occupying octa-interstices $c_{v}^{(0)}=\frac{n_{v}}{N_{A}}$ to be written in the form

$$
c_{v}^{(0)}=\frac{1}{\left(1-c_{C}\right)^{3}} \exp \left\{-\frac{1}{k T}\left(\frac{z}{2} v_{A A}-\sum_{i=1}^{3} c_{C}^{(i)} u_{i}^{(0)}\right)\right\} .
$$

Here $c_{C}$ is the concentration of atoms $C$ occupying octa-interstices in the alloy, $c_{C}^{(i)}$ is the concentration of atoms $C$ occupying interstices of the $i$ th type, $N_{A}$ is the number of central atoms $A$ in the solution, $n_{v}$ is the number of vacancies, $z$ is the coordination number, and $v_{A A}$ is the energy of the pair $A-A$ taken with the minus sign. Considering that the $c_{v}$ value is small, we obtain

$$
\begin{gathered}
\sum_{i=1}^{3} c_{C}^{(i)} u_{i}^{(0)}=3 c_{C} u^{(0)}+\frac{3 c_{C}}{2} a^{2}\left\{\frac{1}{2} j_{A C}\left(\varepsilon_{1}^{2}+\varepsilon_{2}^{2}+\varepsilon_{3}^{2}\right)\right. \\
\left.+j_{A C}^{(1)}\left(\left(\varepsilon_{1}+\varepsilon_{2}\right)^{2}+\left(\varepsilon_{1}+\varepsilon_{3}\right)^{2}+\left(\varepsilon_{2}+\varepsilon_{3}\right)^{2}\right)\right\},
\end{gathered}
$$

from which we finally derive

$$
c_{v}^{(0)}=\frac{1}{\left(1-c_{C}\right)^{3}} e^{-\frac{u^{(0)}}{k T}} \varphi_{1}(T) \varphi_{2}^{(0)}(T),
$$

where $u^{(0)}$ is the energy of vacancy formation in the solution $A-(C)$ with atoms $C$ occupying octa-interstices,

$$
\begin{gathered}
\varphi_{1}(T)=\exp \left\{\frac{1}{k T} \frac{a^{2} j}{12}\left(\varepsilon_{1}+\varepsilon_{2}+\varepsilon_{3}\right)^{2}\right\}, \\
\varphi_{2}^{(0)}(T)=\exp \left\{\frac{3 c_{C} a^{2}}{2 k T}\left[\frac{1}{2} j_{A C}\left(\varepsilon_{1}^{2}+\varepsilon_{2}^{2}+\varepsilon_{3}^{2}\right)+j_{A C}^{(1)}\left(\left(\varepsilon_{1}+\varepsilon_{2}\right)^{2}+\left(\varepsilon_{1}+\varepsilon_{3}\right)^{2}+\left(\varepsilon_{2}+\varepsilon_{3}\right)^{2}\right)\right]\right\}
\end{gathered}
$$

(here $j, j_{A C}$, and $j_{A C}^{(1)}$ are positive constants [3]).

If atoms $C$ occupy tetra-interstices, 


$$
c_{v}^{(T)}=\frac{1}{\left(1-c_{C}\right)^{6}} e^{-\frac{u^{(T)}}{k T}} \varphi_{1}(T) \varphi_{2}^{(T)}(T),
$$

where $u^{(T)}$ is the energy of vacancy formation with atoms $C$ occupying tetra-interstices and

$$
\varphi_{2}^{(T)}(T)=\exp \left\{\frac{3 c_{C} a^{2} j_{A C}}{40 k T}\left[\left(\varepsilon_{1}+4 \varepsilon_{2}\right)^{2}+\left(\varepsilon_{1}+4 \varepsilon_{3}\right)^{2}+\left(\varepsilon_{2}+4 \varepsilon_{1}\right)^{2}+\left(\varepsilon_{2}+4 \varepsilon_{3}\right)^{2}+\left(\varepsilon_{3}+4 \varepsilon_{1}\right)^{2}+\left(\varepsilon_{3}+4 \varepsilon_{2}\right)^{2}\right]\right\} .
$$

For FCC lattices without interstice energy splitting, the vacancy concentration can also be described by Eqs. (8) and (11) in which $1 /\left(1-c_{C}\right)^{3}$ and $1 /\left(1-c_{C}\right)^{6}$ are substituted by $1 /\left(1-c_{C}\right)$ and $1 /\left(1-c_{C}\right)^{2}$ and $\varphi_{2}^{(0)}$ and $\varphi_{2}^{(T)}$ are

$$
\begin{gathered}
\varphi_{2}^{(0)}(T)=\exp \left\{\frac{3 c_{C} j_{A C}^{(0)} a^{2}}{2 k T}\left(\varepsilon_{1}^{2}+\varepsilon_{2}^{2}+\varepsilon_{3}^{2}\right)\right\}, \\
\varphi_{2}^{(T)}(T)=\exp \left\{\frac{3 c_{C} j_{A C}^{(T)} a^{2}}{12 k T}\left(\varepsilon_{1}+\varepsilon_{2}+\varepsilon_{3}\right)^{2}\right\} .
\end{gathered}
$$

In calculations of the diffusion coefficient $D$, we first consider the $A-(C)$ solution with FCC lattice and atoms $C$ occupying octa-interstices.

The height of the diffusion potential barrier is determined by the energy difference between the atom $A$ in position $S$ neighboring to the vacant site $V$ and the saddle point $P$ lying in the middle of the segment $S-V$ [2]:

$$
\Delta U=U(P)-U(S),
$$

where

$$
\begin{gathered}
U(P)=-6 v_{A A}^{0}-6 c_{C} v_{A C}+0.004 j_{A A} a^{2}-0.04 j_{A A} a^{2} c_{C}\left(\varepsilon_{1}+\varepsilon_{2}+\varepsilon_{3}\right)+o\left(\varepsilon^{2}\right), \\
U(S)=-7 v_{A A}^{0}-6 c_{C}\left(v_{A C}+2 v_{A C}^{\prime}\right)+o\left(\varepsilon^{2}\right) .
\end{gathered}
$$

Here $v_{A A}^{0}, v_{A C}$, and $v_{A C}^{\prime}$ are energies of $A-A$ pairs and $A-C$ pairs with the first and second neighbors taken with the minus sign.

From here, neglecting the terms quadratic in $\varepsilon_{i}$, we derive

$$
\Delta U=v_{A A}^{0}+0.004 j_{A A} a^{2}+12 c_{C} v_{A C}^{\prime}-0.04 j_{A A} a^{2} c_{C}\left(\varepsilon_{1}+\varepsilon_{2}+\varepsilon_{3}\right) .
$$

In the approximation linear in the diffusion tensor components, substituting Eq. (18) into Eq. (4), we finally obtain

$$
D_{i}=\frac{1+2 \varepsilon_{i}}{\left(1-c_{C}\right)^{3}} \frac{a^{2}}{\tau_{0}} e^{-\frac{Q_{0}+\Delta Q}{k T}}
$$

where $Q_{0}=\Delta U_{0}+u_{v}$ ( $u_{v}$ is the energy of vacancy formation in the non-deformed $A-(C)$ crystal) is the energy of selfdiffusion activation in the non-deformed solution and $\Delta Q$ is its change after deformation: 


$$
\Delta Q=a\left(\varepsilon_{1}+\varepsilon_{2}+\varepsilon_{3}\right), \quad a=\text { const }
$$

This additive term $\Delta Q$ in Eq. (19) is proportional to $\varepsilon_{1}+\varepsilon_{2}+\varepsilon_{3}=\frac{\Delta V}{V}-$ relative change of the volume. Deformation generally leads to small anisotropy $D$ (of the order of several percent). We now pay attention to the strong dependence of $D$ on the impurity concentration. Thus, $D$ increases approximately by $3 \%$ for $c_{C} \approx 0.01$ and by $16 \%$ for $c_{C} \approx 0.05$.

Analogous result are obtained for the $A-(C)$ solutions with atoms $C$ incorporated in tetra-interstices and the only difference that the term $1 /\left(1-c_{C}\right)^{6}$ with stronger dependence of $D$ on the impurity concentration arises in the preexponential factor of $D$ instead of the term $1 /\left(1-c_{C}\right)^{3}$.

A more complicated situation is observed for alloys with FCC lattice. The diffusion flux in the direction of the $O x$ axis in this case arises due to transitions of central atoms to the neighboring vacant sites located in planes perpendicular to the $O z$ and $O y$ axes, so that

$$
j_{x}=j_{x}^{(1)}+j_{x}^{(2)} .
$$

In the first case, considering that atoms $C$ occupy octa-interstices, we perform calculations analogous to Eqs. (15)-(18) and obtain that the height of the diffusion potential barrier is

$$
\Delta U=7 v_{A A}^{0}+4 v_{A C}^{0}+\Gamma a^{2}\left(\varepsilon_{1}+\varepsilon_{2}+4 \varepsilon_{3}\right),
$$

where

$$
\Gamma=2 j_{A A}+c_{C} j_{A C} .
$$

In the second case, the $\Delta U$ value differs by substitutions $\varepsilon_{2} \rightarrow 4 \varepsilon_{2}$ and $4 \varepsilon_{3} \rightarrow \varepsilon_{3}$.

Calculating fluxes $j_{x}^{(1)}$ and $j_{x}^{(2)}$ and considering that $\varepsilon_{i}$ are small, we obtain

$$
D_{x}=\frac{1+2 \varepsilon_{1}}{1-c_{C}} \frac{a^{2}}{\tau_{0}} \exp \left(-\frac{Q_{0}+\Delta Q_{x}}{k T}\right)
$$

where

$$
\Delta Q_{x}=\frac{\Gamma a^{2}}{2}\left(\varepsilon_{1}+5 \varepsilon_{2}+5 \varepsilon_{3}\right)
$$

Expressions for $D_{y}$ and $D_{z}$ are derived from Eq. (24) in which $\varepsilon_{1}$ in the pre-exponential factor and in $\Delta Q_{x}$ has been replaced by $\varepsilon_{2}$ and $\varepsilon_{3}$, respectively.

Thus, unlike the BCC crystals, the anisotropy of not only the pre-exponential factor, but also of the selfdiffusion activation energy is observed in FCC solutions. Analogous results are obtained for diffusion in solutions with impurity atoms occupying tetra-interstices; in this case, the factor $1 /\left(1-c_{C}\right)^{2}$ stands for the factor $1 /\left(1-c_{C}\right)$ in the expression for $D$.

The author considers it his pleasant duty to express his gratitude to Professor S. I. Masharov for useful discussions of the results obtained in this work. 


\section{REFERENCES}

1. A. A. Smirnov, Molecular Theory of Metals [in Russian], Nauka, Moscow (1966).

2. A. A. Smirnov, The Theory of Interstitial Alloys [in Russian], Nauka, Moscow (1979).

3. V. A. Volkov and S. I. Masharov, Russ. Phys. J., 54, No. 12, 1406-1412 (2012).

4. V. A. Volkov, G. S. Masharov, and S. I. Masharov, Fiz. Met. Metalloved, 102, No. 3, 261-263 (2006). 